\title{
Current Situation and Hotspot of Domestic Agricultural Product Brand Research Visual Analysis Based on Bibliometric Analysis
}

\author{
Gao Kai ${ }^{1}$, a ,Chen Jiaying ${ }^{2}$, ${ }^{*}$, Liu Yimin ${ }^{3 \mathrm{c}}$ \\ ${ }^{1}$ Business school of sichuan agricultural university, Chengdu 611830, China \\ ${ }^{2}$ Business school of sichuan agricultural university, Chengdu 611830, China \\ ${ }^{3}$ Business school of sichuan agricultural university, Chengdu 611830, China \\ a1016941246@qq.com \\ ${ }^{b^{*}}$ cjy@sicau.edu.cn \\ c307837611@qq.com
}

\begin{abstract}
To understand the domestic research present situation and the hotspot in t-he field of agricultural pro ducts brand, adopting the method of literature metrology, com-prehensive utilization of NoteExpress, SPSS, Ucient, Netdraw software, such as making a map visualization, and further research in the fiel $\mathrm{d}$ of cluster analysis, multidimensional scaling analysis and social network analysis, in order to more accurately grasp the main research direction in the field of domestic agricultural products brand rese arch. The results show that the research field of agricultural products brand in China involves six ca tegories, including regional brand of agricultural products, marketing strategy of green agricultural pro ducts, marketing strategy of agricultural products and branding strategy of agricultural products. Resea rch hotspots include "geographical indications", "agricultural products brands", "regional agricultural pr oducts brands", "brand building", "characteristic agricultural products" and other research directions.

Keywords: Agricultural products brand, Visual analysis, Cluster analysis, Social network analysis
\end{abstract}

\section{国内农产品品牌领域研究的现状与热点 基于文献计量的可视化分析 高凯 ${ }^{1,2}$, 陈佳莹 ${ }^{2, b e}$, 刘宜敏 ${ }^{3.4}$}

${ }^{1}$ 四川农业大学商学院, 四川成都, 611830

${ }^{2}$ 四川农业大学商学院, 四川成都, 611830

${ }^{3}$ 四川农业大学商学院, 四川成都, 611830

a1016941246@qq.com

$b^{*}$ cjy@sicau.edu.cn

c307837611@qq.com

\section{摘要}

为了解国内农产品品牌领域的研究现状与热点, 采用文献计量的分析方法, 综合利用 NoteExpress、 SPSS、Ucient、Netdraw 等软件制作出可视化图谱, 并进一步对该领域的研究进行聚类分析、多维尺 度分析和社会网络分析, 以较为准确地把握国内农产品品牌研究领域的主要研究方向。结果表明, 我 国农产品品牌研究领域涉及乡村振兴下的农产品区域品牌、绿色农产品营销战略、农产品品牌营销策 略、农产品品牌化战略等六大类别; 研究热点涵盖 “地理标志”、“农产品品牌”、“农产品区域品牌”、 “品牌建设”、“特色农产品” 等研究方向。

关键词: 农产品品牌; 可视化分析; 聚类分析; 社会网络分析 


\section{1. 前言}

在现如今农产品激励的市场竞争中, 农产品不再依 靠传统的价格策略, 转而逐渐走向品牌化的道路。2018 年中央 1 号文件提出质量兴农之路, 同年六月农业农村 部发布《关于加快推进品牌强农的意见》, 文件指出深 入推进品牌强农是经济高质量发展的迫切要求, 是推进 农业供给侧结构性改革的现实路径, 是提升农业竞争力 的必然选择, 是促进农民增收的有力举措 ${ }^{[1]}$ 。而在学术 研究领域, 国内关于农产品品牌领域的研究多以单个研 究主题进行分散的独立研究, 而少有对该领域研究现状 进行可视化的系统梳理。鉴于此, 本文利用可视化技术, 并结合系统聚类分析、多维尺度分析、社会化网络分析 等方法清晰的展现国内农产品品牌领域的研究现状与 热点, 以期为该领域的研究提供学习参考价值。

\section{2. 数据和研究方法}

\section{1. 样本采集}

本文以中国知网数据库作为数据源, 文献类型选择 “期刊”, 检索主题设置为 “农产品品牌”, 检索时间范 围为 2010 - 2019 年, 为保证样本数据的质量与代表性, 来源类别选择核心期刊与 CSSCI, 共得到相关文献 381 篇, 经剔除新闻通讯、会谈纪要等不相关文献后, 最终 得到有效研究样本 360 篇期刊文献。

\section{2. 研究方法}

本研究主要采用文献计量分析方法, 对国内农产品 品牌领域的发文时间分布、作者及机构合作、期刊分布 等研究现状情况进行基础统计分析, 并综合利用 NoteExpress、SPSS、Ucient、Netdraw 等软件制作出可 视化图谱, 进一步对该领域的研究进行聚类分析、多维 尺度分析和社会网络分析, 以较为准确地把握国内农产 品品牌研究领域的主要研究方向。

\section{3. 农产品品牌领域研究现状}

\section{1. 文献时间分布}

本文根据 CNKI 数据库导出的文献数据, 对 2010-2019 年期间农产品品牌研究领域的文献时间分布 情况进行统计, 并制作出发文年代分布图 (如图 1)。从 图中可以看出, 该领域近十年文献发文数量波动较大, 而根据数据统计结果, 该领域近十年间共计发表文献数 量 360 篇, 年均发文量为 36 篇, 历年发文基本呈平稳
态势, 说明学者对农产品品牌领域的研究持续保持关注。

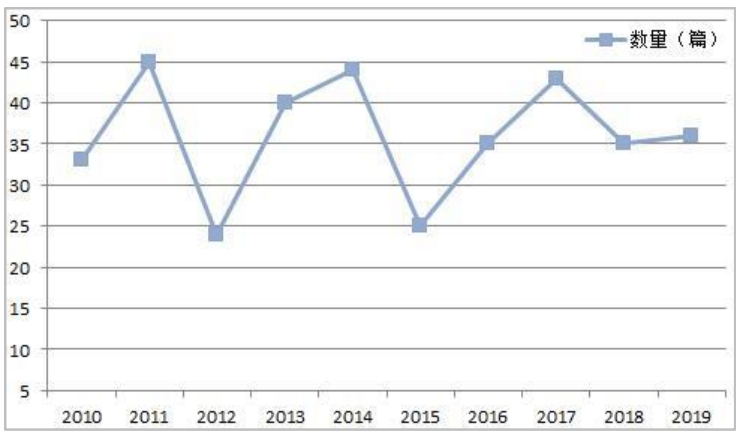

图 1 2010-2019 年农产品品牌研究文献时间分布

\section{2. 高产作者合作情况}

对高产作者进行共现分析不仅可以发现该领域内 活跃的核心研究作者, 还可以反映出研究作者及机构之 间的相互合作程度。图 2 列出了农产品品牌领域发文量 排名前 10 位的研究者, 根据数据统计, 该领域内发文 数量最高的是李艳军 8 篇, 仅占总发文量的 $2.22 \%$, 而 排名第十位只有 3 篇。在作者合作关系网络图中，节点 大小表示作者发文量，同一节点颜色表示作者所处同一 机构, 节点之间的连线表示作者及机构之间的相互合作 关系。从图可知, 在排名前十位的作者合作关系网络中, 作者及机构之间的合作关系非常分散，图中仅有两对合 作关系, 且仅有一组为跨机构合作。据此说明, 虽然农 产品品牌领域的研究持续受到学者的关注, 但进行持续 深入研究的学者还比较少, 且整个合作研究体系还比较 薄弱, 研究者之间的合作程度并不高, 跨机构合作还需 进一步加强。

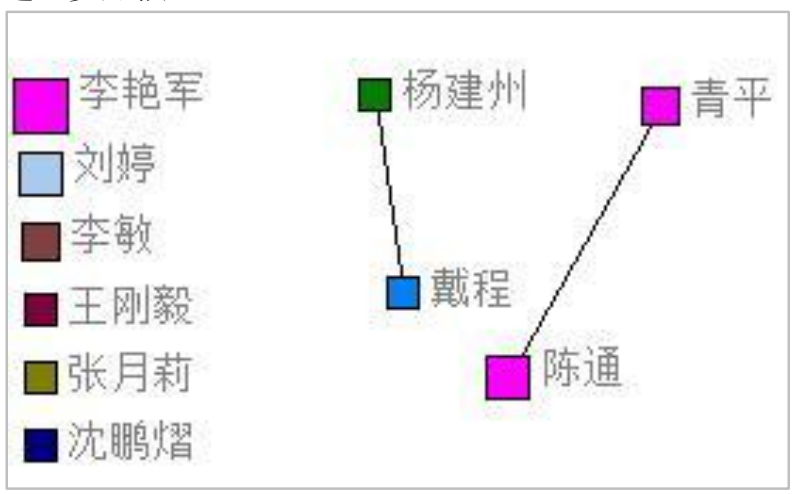

图 2 高频作者合作关系网络

\section{3. 主要来源期刊分布}

通过对主要来源期刊的分布情况进行分析, 可以掌 握农产品品牌研究受到哪些领域的重点关注。表 1 展现 了该领域内载文量排名前 10 位的主要来源期刊分布情 况, 从载文数量上看, 《农业经济》刊文量最高, 占总 文献比例 $12.78 \%$, 超过年均发文量 36 篇的水平，是该 领域内期刊发文的核心力量。而从期刊类型上说，前 10 
名期刊类型包括了农业经济、农业科学以及商业商贸等, 也反映出农产品品牌的研究除了受到农业经济、农业科
学等在农业领域重点关注之外，也是商业商贸等方面的 关注领域。

表 1 主要来源期刊分布情况（top10）

\begin{tabular}{ccc|ccc}
\hline 期刊名称 & 载文量 & 占总比 & 期刊名称 & 载文量 & 占总比 \\
\hline 农业经济 & 46 & $12.78 \%$ & 安徽农业科学 & 12 & $3.33 \%$ \\
商业经济研究 & 26 & $7.22 \%$ & 商业时代 & 10 & $2.78 \%$ \\
江苏农业科学 & 13 & $3.61 \%$ & 价格月刊 & 9 & $2.50 \%$ \\
中国农业资源与区划 & 12 & $3.33 \%$ & 农业经济问题 & 9 & $2.50 \%$ \\
世界农业 & 12 & $3.33 \%$ & 中国商贸 & 8 & $2.22 \%$ \\
\hline
\end{tabular}

\section{4. 农产品品牌领域研究热点}

\section{1 . 关键词分析}

关键词是一篇文章核心内容的浓缩和提炼，如果某 一关键词在其所在领域的文献中反复出现, 则反映出该
关键词所表征的研究主题是该领域的研究热点 ${ }^{[2]}$ 。通过 文献检索与管理系统 NoteExpress 软件数据分析功能对 CNKI 数据库导出的 360 篇文献提取关键词, 共得到关键 词 783 个, 为了使关键词更具有分析价值, 本研究选取 了排名前 30 的高频关键词进行分析。如表 2 所示，通 过这些关键词的频次能初步判断它们在农产品品牌研 究领域中的主要研究方向。

表 2 关键词列表（top30）

\begin{tabular}{ccc|ccc|ccc}
\hline 序号 & 关键词 & 频次 & 序号 & 关键词 & 频次 & 序号 & 关键词 & 频次 \\
\hline 1 & 农产品 & 92 & 11 & 品牌营销 & 11 & 21 & 品牌战略 & 7 \\
2 & 农产品品牌 & 35 & 12 & 绿色农产品 & 10 & 22 & 策略 & 7 \\
3 & 区域品牌 & 30 & 13 & 农业 & 9 & 23 & 营销策略 & 7 \\
4 & 特色农产品 & 25 & 14 & 对策 & 9 & 24 & 竞争力 & 7 \\
5 & 品牌 & 24 & 15 & 乡村振兴 & 9 & 25 & 家庭农场 & 6 \\
6 & 品牌建设 & 21 & 16 & 电子商务 & 8 & 26 & 日本 & 6 \\
7 & 农产品区域品牌 & 19 & 17 & 特色农业 & 8 & 27 & 结构方程模型 & 6 \\
8 & 地理标志 & 13 & 18 & 农业品牌 & 8 & 28 & 问题 & 6 \\
9 & 品牌竞争力 & 12 & 19 & 影响因素 & 8 & 29 & 品牌价值 & 6 \\
10 & 农民专业合作社 & 11 & 20 & 营销 & 7 & 30 & 品牌塑造 & 5 \\
\hline \multicolumn{7}{r}{} \\
\multicolumn{7}{r}{} & \multicolumn{5}{c}{} & &
\end{tabular}

\section{2. 关键词共现矩阵与相异矩阵}

为便于开展多维尺度分析及社会网络分析需要, 利 用 NoteExpress 软件对高频关键词列表进行转换, 分别 构建出高频关键词共现矩阵 (表 3 ) 与相异矩阵 (表 4)。
在关键词共现矩阵中, 其对角线数值代表两两关键词同 时出现的频数; 而在相异矩阵中，对角线数值代表两两 关键词的相关性, 数值越接近于 1 , 表示两者的相关性 越低, 反之则两者关联性越强。

表 3 高频关键词共现矩阵（部分）

\begin{tabular}{ccccccc}
\hline 关键词 & 农产品 & 农产品品牌 & 区域品牌 & 特色农产品 & 品牌 & 品牌建设 \\
\hline 农产品 & 92 & 1 & 15 & 0 & 9 & 5 \\
农产品品牌 & 1 & 35 & 0 & 0 & 0 & 2 \\
区域品牌 & 15 & 0 & 30 & 2 & 0 & 1
\end{tabular}




\begin{tabular}{ccccccc} 
特色农产品 & 0 & 0 & 2 & 25 & 2 & 3 \\
品牌 & 9 & 0 & 0 & 2 & 24 & 0 \\
品牌建设 & 5 & 2 & 1 & 3 & 0 & 21 \\
\hline
\end{tabular}

表 4 高频关键词相异矩阵（部分）

\begin{tabular}{ccccccc}
\hline 关键词 & 农产品 & 农产品品牌 & 区域品牌 & 特色农产品 & 品牌 & 品牌建设 \\
\hline 农产品 & 0 & 0.9997 & 0.9185 & 1 & 0.9633 & 0.9871 \\
农产品品牌 & 0.9997 & 0 & 1 & 1 & 1 & 0.9946 \\
区域品牌 & 0.9185 & 1 & 0 & 0.9947 & 1 & 0.9984 \\
特色农产品 & 1 & 1 & 0.9947 & 0 & 0.9933 & 0.9829 \\
品牌 & 0.9633 & 1 & 1 & 0.9933 & 0 & 1 \\
品牌建设 & 0.9871 & 0.9946 & 0.9984 & 0.9829 & 1 & 0 \\
\hline
\end{tabular}

\section{3. 聚类分析}

聚类分析可以根据多个变量之间的关系远近将关 键词进行分类, 更有助于系统理解农产品品牌领域研究 的主要类别。将上述高频关键词相异矩阵导入 SPSS 软 件, 利用其系统聚类功能, 绘制出系统聚类谱系图, 图 中纵轴表示的是关键词的相似程度。如图 3 所示, 依据 关键词之间的相似性, 农产品品牌领域的研究热点被分 为六大类别。

（1）农民专业合作社下的农产品品牌建设。该类 别包括农民专业合作社、品牌建设、特色农产品、对策、 问题、策略六个关键词。在农村合作经济组织中，农民 专业合作社既是当地特色农产品的生产者, 同时也是农 产品销售经营环节的主力军。尽管很多农民专业合作社 拥有了自己的产品品牌, 但是缺乏品牌内涵的现象也很 突出, 所以农民专业合作社的成长离不开品牌建设 ${ }^{[3]}$ 。 在学术研究领域, 研究者主要是对西部地区的农民专业 合作社现存问题及应对策略进行了探讨, 田文勇 ${ }^{[4]}$ 等学 者基于四川、贵州两地的调查提出应从农产品生产、销 售以及合作社内部认识等方面推进品牌建设, 而鲜阳红 ${ }^{[5]}$ 则针对西部民族地区的特色农产品品牌构建还需加大 国家政策性扶持。

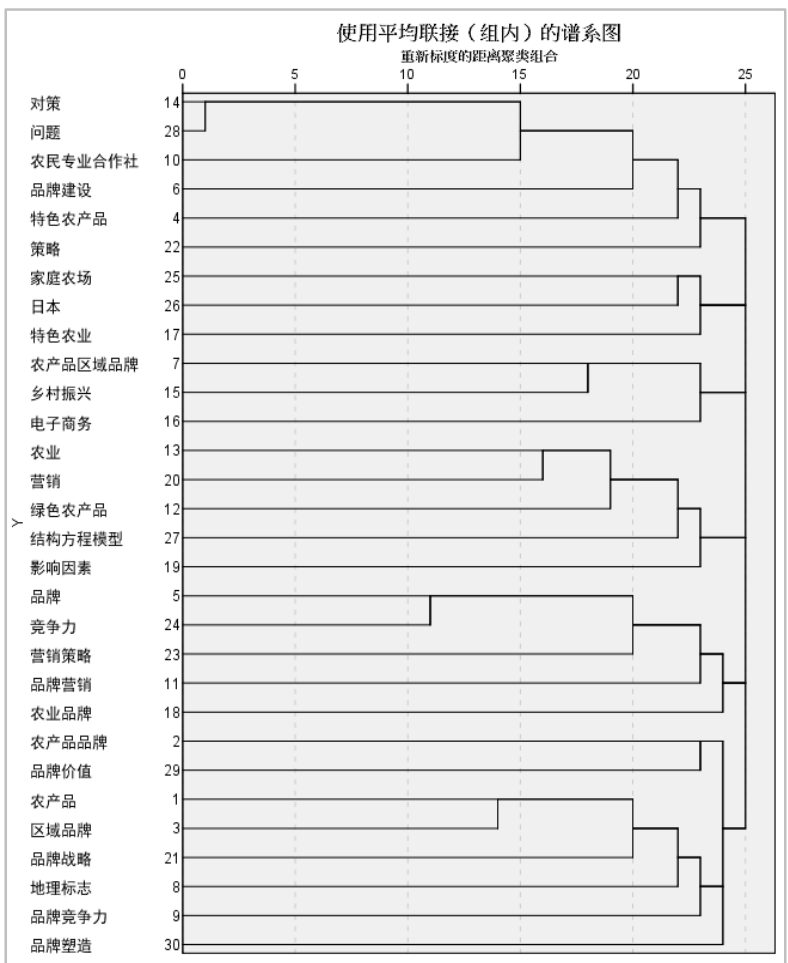

图 3 共词矩阵系统聚类

（2）日本农产品品牌对中国的启示。该类别包括 日本、家庭农场、特色农业三个关键词。我国农产品品 牌起步较晚, 与发达国家相比还存在明显差距, 因此部 分学者展开了对国外发达国家的研究, 其中以日本为代 表的研究较为突出。孙华美结合美日经验, 提出加快中 国特色农产品品牌发展的对策应从政府扶持、质量监管、 品牌宣传、科技研发等方面入手 ${ }^{[6]}$ 。张文超 ${ }^{[7]}$ 以日本农 产品营销经验展开研究, 提出中国特色农业营销路径选 择还应该关注品牌保护和明确市场定位等。

（3）乡村振兴下的农产品区域品牌。该分类下包 括乡村振兴、农产品区域品牌以及电子商务三个关键词。 创建与培育农产品区域品牌是贯彻落实乡村振兴战略 的重要途径, 是实施质量兴农战略规划的关键问题 ${ }^{[8]}$ 。作 为乡村振兴战略的重要举措, 学者对此高度关注, 研究 
内容涉及影响因素、运行机制、发展策略等, 案例研究 包括河南、福建、陕西、湖北等省份, 研究视角包含了 乡村振兴战略、产业融合、产业集群、消费者等视角。 另外, 还有部分学者对于农产品电商品牌化 ${ }^{[9]}$ 以及品牌 培育对农产品电商发展的影响 ${ }^{[10]}$ 展开了研究。

（4）绿色农产品营销战略。该类别下包括农业、 营销、绿色农产品、结构方程模型、影响因素五个关键 词。绿色农产品营销旨在通过对农产品进行全程研发、 生产、销售等, 以此来满足消费者对农产品的健康需求 [11]。但是, 在实施绿色农产品营销的过程中, 尚且面临一 些制约因素, 因此学术界也对该领域进行了探讨。绿色 营销的前提是绿色生产, 徐姝 ${ }^{[12]}$ 等学者运用结构方程模 型, 对消费者对农产品可追溯体系接受意愿的影响因素 进行了研究, 王瑛则认为绿色农产品营销应从优化产品、 价格、渠道和促销战略等方面着手, 走差异化品牌发展 道路 ${ }^{[13]}$ 。

（5）农产品品牌营销策略。该分类包括了品牌、 竞争力、营销策略、品牌营销、农业品牌五个关键词。 在农产品激烈的市场竞争中，如若没有品牌营销是万万 不行的。近年来, 国内农产品品牌营销存在着品牌意识 薄弱、营销思维固化等问题, 严重影响了我国农产品带 来的经济利益。对于此, 诸多学者针对农产品品牌营销 展开研究, 如 “互联网+” 时代、新媒体时代、网络社 群等不同视角的营销策略研究, 与此同时, 还对农业品 牌建设及农业现代化的农业品牌政策进行了探讨。孙强 等提出推进中国农业现代化, 应加强农业品牌顶层制度
设计、完善制度框架、强化政策支持，率先推进中国农 业品牌现代化 ${ }^{[14]}$ 。

（6）农产品品牌化战略。该分类下包括了农产品 品牌、品牌价值、农产品、区域品牌、地理标志、品牌 战略、品牌竞争力、品牌塑造八个关键词。该类别占据 排名前 30 关键词的 $26.67 \%$, 表明该类别的研究在农产 品品牌领域中占据了重要地位。农产品品牌化是现代农 业发展的必然趋势, 在农产品品牌化战略的研究中, 学 者较多的关注农产品品牌化的重要性以及实现路径。许 文苹 ${ }^{[15]}$ 等利用实证分析法阐释实现我国地理标志农产 品品牌化的重要意义, 连奕昕 ${ }^{[16]}$ 使用 SWOT 分析法, 并 结合 AHP 层次分析法, 确定当前我国农产品品牌化发展 应采取积极的 SO 扩张型战略。

\section{4. 多维尺度分析}

多维尺度分析能够通过降维的方法, 衡量变量之间 的距离, 反映两点间相似和相异程度, 识别数据间的结 构关系 ${ }^{[17]}$ 。通过将高频关键词相异矩阵 (表 4) 导入到 SPSS 软件后, 选择分析菜单下多维标度功能绘制出农产 品品牌研究领域的坐标图 (图 4)。图中 X 轴为向心度 (Centrality)，表示研究领域之间相互影响程度，向 心度越大，表明越核心； Y 轴为密度 (Density), 表示 研究领域内部之间联系程度, 密度越大, 表明越成熟 ${ }^{[18]}$ 。

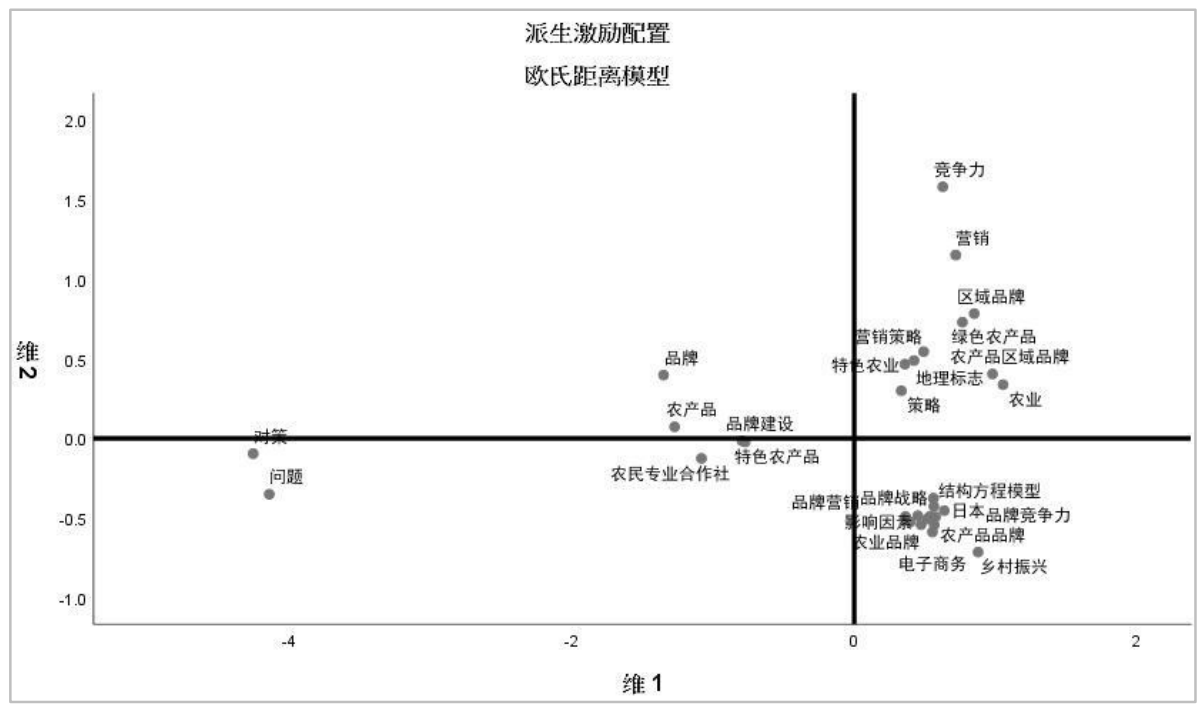

图 4 多维尺度分析坐标图

从图 4 可知, 多维尺度分析坐标图中除了农民专业 合作社下的农产品品牌建设与日本农产品品牌对中国 的启示研究领域以外, 其它四大研究领域从整体上看都 拥有较好的向心度和密度, 说明国内农产品品牌领域的 研究较为成熟和稳定。但是在各大领域内部, 研究密度 还较为分散, 且领域内的研究尚不够成熟, 内部联系程 度较差。而在农民专业合作社下的农产品品牌建设与日
本农产品品牌对中国的启示的研究领域中, 虽然部分关 键词拥有较好的向心度，但 “对策” 和 “领域” 等关键 词处于坐标边缘位置, 内部研究整体密度都较低, 说明 这两个领域尽管都保持较高的关注度，但是仍有部分研 究正在向其他领域过渡。 


\section{5. 社会网络分析}

为进一步探究关键词之间的社会网络关系, 本文基 于高频关键词共现矩阵数据 (表 3), 并借助 Ucinet 与 Netdraw 软件制图功能, 绘制出高频关键词共词网络 (图 5)。图中, 节点的大小代表关键词的共现频次, 而节点 所处的位置表示该节点在网络图中的地位。
点度中心度是研究网络图中节点权力和影响力的 重要指标, 分为绝对度中心度和相对度中心度, 点度中 心度越高, 表明在网络中越占有中心地位 ${ }^{[19]}$ 。利用 Ucinet 软件对高频关键词共词矩阵执行 CentralityDegree 命令, 得到点度中心度数据（表 4)。表中点度 中心度越大, 表明其影响力越大, 所处的地位就越核心。

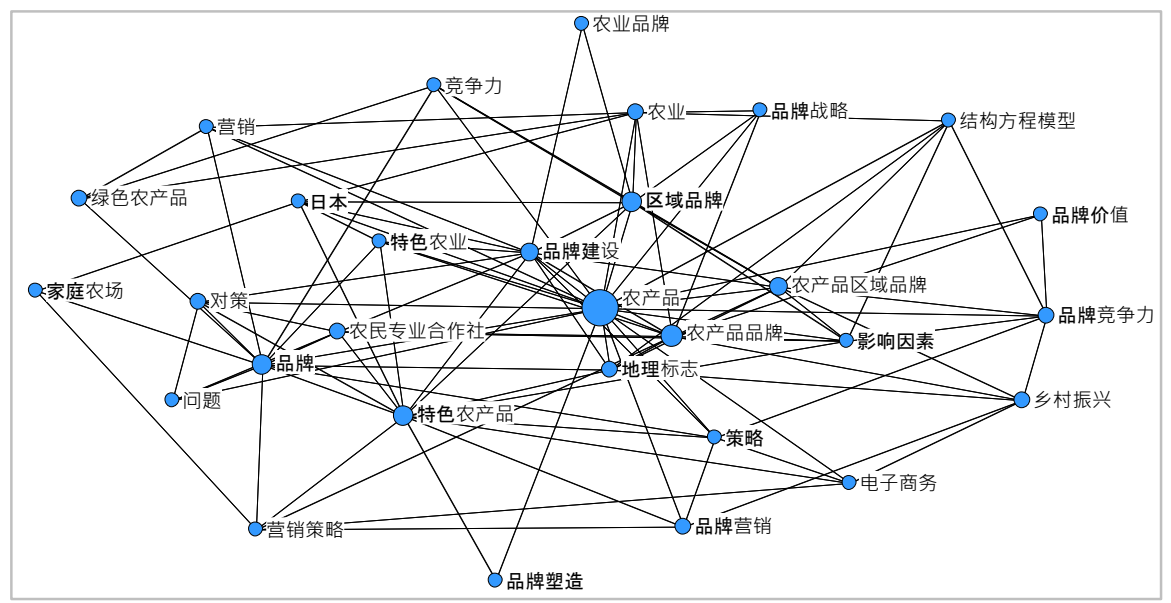

图 5 共词网络图

表 5 高频关键词度数中心度

\begin{tabular}{ccc|ccc|ccc}
\hline 关键词 & $\mathrm{D}$ & $\mathrm{nD}$ & 关键词 & $\mathrm{D}$ & $\mathrm{nD}$ & 关键词 & $\mathrm{D}$ & $\mathrm{nD}$ \\
\hline 农产品 & 81 & 0.19 & 品牌营销 & 10 & 0.02 & 品牌战略 & 9 & 0.02 \\
农产品品牌 & 17 & 0.04 & 绿色农产品 & 6 & 0.01 & 策略 & 9 & 0.02 \\
区域品牌 & 26 & 0.06 & 农业 & 10 & 0.02 & 营销策略 & 8 & 0.02 \\
特色农产品 & 20 & 0.05 & 对策 & 13 & 0.03 & 竞争力 & 9 & 0.02 \\
品牌 & 29 & 0.07 & 乡村振兴 & 8 & 0.02 & 家庭农场 & 3 & 0.01 \\
品牌建设 & 21 & 0.05 & 电子商务 & 5 & 0.01 & 日本 & 9 & 0.02 \\
农产品区域品牌 & 14 & 0.03 & 特色农业 & 6 & 0.01 & 结构方程模型 & 6 & 0.01 \\
地理标志 & 16 & 0.04 & 农业品牌 & 2 & 0.00 & 问题 & 7 & 0.02 \\
品牌竞争力 & 12 & 0.03 & 影响因素 & 8 & 0.02 & 品牌价值 & 6 & 0.01 \\
农民专业合作社 & 10 & 0.02 & 营销 & 10 & 0.02 & 品牌塑造 & 4 & 0.01 \\
\hline
\end{tabular}

现结合图 5 网络结构和表 5 数据结果来看, 关键词 “农产品” 的点度中心度最高, 它处于整个网络中的最 核心位置, 表明该领域内的研究都是围绕它进行展开的。 在处于次核心地带的以“地理标志”、“农产品品牌”、“农 产品区域品牌”、“品牌建设” 为代表的关键词中, 尽管 他们没有处于网络的核心, 但是他们都拥有较高的点度 中心度, 同样是农产品品牌领域研究的热点。除此之外, 以“区域品牌”、“特色农产品”、“品牌” 等为代表的关 键词, 虽然他们处于网络中相对较远位置, 但是他们的 点度中心度较高, 说明他们在各自的领域中同样发挥着 重要作用。而位于网络边缘位置的关键词, 以“品牌塑 造”、“品牌价值”、“家庭农场”、“农业品牌”、“乡村振
兴” 等为代表，表明该领域的研究正逐渐多元化，衍生 出了在不同领域之间相结合的研究, 但在该方面的研究 还相对不足, 未来的研究的空间还很大。

\section{5. 结论及不足}

本文通过对国内农产品品牌的 360 篇文献进行聚类 分析、多维尺度分析和社会网络分析等文献计量分析方 法, 最后得出以下结论:

（1）在 2010-2019 年期间，国内农产品品牌领域 的研究基本保持平稳态势, 学术界对该领域的研究保持 持续关注, 但进行深入研究的学者还比较少。同时, 整 
个作者及机构的合作研究体系较为薄弱, 研究者之间合 作程度不高, 跨机构合作还需进一步加强。

（2）通过系统聚类分析，将国内农产品品牌领域 的研究分为了农民专业合作社下的农产品品牌建设、日 本农产品品牌对中国的启示、乡村振兴下的农产品区域 品牌、绿色农产品营销战略、农产品品牌营销策略、农 产品品牌化战略六大类别。结合多维尺度分析后得出, 乡村振兴下的农产品区域品牌、绿色农产品营销战略、 农产品品牌营销策略、农产品品牌化战略这四个领域都 拥有较好的向心度和密度, 说明国内农产品品牌领域的 研究基本成熟和稳定, 但是在各大热点领域内部, 都还 存在研究密度分散, 内部联系程度较差的特点。

(3) 通过社会网络分析, 国内农产品品牌领域的 研究以 “农产品” 为焦点进行展开, 同时发现在整个研 究领域内 “地理标志”、“农产品品牌”、“农产品区域品 牌”、“品牌建设”、“特色农产品” 等为代表的关键词已 成为该领域的研究热点。另外, 在该领域内以 “家庭农 场”、乡村振兴” 等为代表的多元化的研究正在逐渐形 成，值得未来进一步探索。

本研究只选取了近 10 年间国内农产品品牌研究领 域的 360 篇期刊文献, 没有对所有文献进行统计分析, 使得对该领域的研究现状与热点分析不够完整, 同时也 缺少对发展趋势的分析。此外, 本研究没有加入国际刊 物的研究成果, 后续研究可从上述问题进行考虑, 以补 不足。

\section{REFERENCES}

[1] Ministry of Agriculture and Rural Affairs.(2018)O pinions on Accelerating Brand Promotion to Strengthe n Agriculture (Nongshifa [2018] No.3).http://www.moa. gov.cn/nybgb/2018/201807/201809/t20180912_6157133.h tm

[2] Zhao,R.Y., Guo,F.J.,Zhao,Y.H. (2015) Mainstream research field and hot frontier research of scientometri cs .J. Books and information work,59(02):66-74.

[3] Wang,Y. (2013) Experience, problems and counter measures for brand building of farmers' professional $\mathrm{c}$ ooperatives. J. Agricultural mechanization research,35(1 1):9-12.

[4] Tian,W.Y., Zhao,S.W., Zhang,P. (2014) Cooperativ e factors affecting the empirical study on the construct ion of agricultural products brand, based on the part o f guizhou, sichuan farmers' professional co-operatives $\mathrm{s}$ urvey .J. Journal of development studies,(5):30-33.

[5] Xian,Y.H. (2014) Brand construction of featured agricultural products of farmers' specialized cooperative $\mathrm{s}$ in western ethnic areas .J. Guizhou ethnic studies,35 (05):140-143.

[6] Sun,H.M. (2016) Brand Building Strategies for A gricultural Products with Chinese Characteristics based on the Us-Japan Experience .J. World Agriculture,(0 6):36-39.

[7] Zhang,W.C. (2017) Agricultural Product Marketin g Experience of Japanese "brand Agriculture" and Agr icultural Path Selection with Chinese Characteristics .J. World Agriculture,(06):173-176.

[8] Xu,M. (2019) Cultivation Path of Regional Agric ultural Products Brand in Jilin Province under the Bac kground of Rural Revitalization Strategy .J. Taxation a nd Economy,(06):106-110.

[9] Zhang,Y.T., LI,D. (2016) Research on the influen ce path of origin image on e-commerce Branding of a gricultural products .J. China Soft Science,(05):43-54.

[10] Lu,Z.Y. (2018) Research on the Impact of Brand Cultivation on the Development of Agricultural Produ cts E-commerce -- Based on 2,131 questionnaires sent to 15 provincial units in the east, Central and West of China .J. Modern Economics Discussion,(02):87-99.

[11] Tian,J. (2015) Concept of Promoting Sustainable Agricultural Development with green Agricultural Prod uct Marketing .J. Agricultural Economy,(06):116-118.

[12] Xu,S., X,K., CAI,Y. (2019) Research on factors i nfluencing consumers' Willingness to accept Agricultur al Products traceability System -- From the perspective of Extended Technology Acceptance Model .J. Journ al of Guizhou University of Finance and Economics, $(0$ 1):82-92

[13] Wang,Y. (2019) Research on brand Positioning an d Marketing Strategy Optimization of Green Agricultur al Products from the Perspective of Green Production J. Agricultural Economy,(08):127-129.

[14] Sun,Q., Zhong,Y.L. (2019) Research on Chinese agricultural brand policy towards agricultural moderniz ation .J. Hebei journal,39(01):130-136.

[15] Xu,W.P., Chen,T. (2011) Analysis on the necessit $\mathrm{y}$ of branding agricultural products with geographical i ndications in China .J. Journal of tianjin university (so cial science edition),13(04):303-307.

[16] Lian,Y.X., Xu,J., LI,X.Z. (2017) SWOT AHP An alysis and Countermeasures of Agricultural Product $\mathrm{Br}$ anding Development .J. Commercial And Economic R esearch,(16):131-133.

[17] Chen,H.X., Guan,C., Wang,Y.G.X. (2020) Current situation and hot spots of domestic research on transf ormation of scientific and technological achievements - from the perspective of co-word analysis and social network analysis .J. Science and technology manageme 
nt research,40(07):125-134.

[18] Li,Y.Y. (2016) Research hotspots and content anal ysis in the field of "Internet + " in China .J. Journal o $\mathrm{f}$ intelligence,35(8):128-132.

[19] Liu,J. (2014) Overall Network Analysis.Shanghai: Shanghai People's Publishing House,Shanghai. 